ENSAIO

Recebido em:

$31 / 05 / 2016$

Aceito em:

$12 / 08 / 2016$

\title{
Acoplamento bibliográfico e análise de cocitação: revisão teórico-conceitual
}

\author{
Bibliographic coupling and co-citation analysis: theoretical- \\ conceptual review
}

\section{Maria Cláudia Cabrini GRÁcIO}

Professora do Departamento de Ciência da Informação - UNESP - cabrini@marilia.unesp.br

\section{Resumo}

A análise relacional de citação, realizada sobretudo pelos métodos de cocitação e de acoplamento bibliográfico, permite conhecer as relações estruturais de conectividade teórico-metodológica de um domínio, a proximidade, a vizinhança, a associação e a interlocução estabelecida entre documentos e pesquisadores, entre outros, como reconhecidos pela comunidade científica. Esses dois métodos têm sido utilizados, individualmente, para a compreensão da comunicação científica, a frente de pesquisa e a estrutura intelectual de domínios científicos. Recentemente, tem se observado estudos comparativos entre eles a fim de verificar suas adequações e eficiência como indicadores de similaridade ou proximidade temática. Neste contexto, dada a importância desses métodos para as análises bibliométricas e de domínio, este estudo tem por objetivo discutir aspectos teóricos e conceituais inerentes aos dois métodos de análise das relações entre citações, Análise de Cocitação de Autores e Análise de Acoplamento Bibliográfico de Autor, visando contribuir para as reflexões e desenvolvimento dos estudos teórico-metodológico brasileiros na temática.

Palavras-chave: Análise de cocitação. Acoplamento bibliográfico. Análise relacional de citação. Análise de domínio.

\begin{abstract}
The relational citation analysis, mainly co-citation method and bibliographic coupling, allows to know the structural relations of theoretical and methodological connectivity of a domain, as well as the proximity, the neighborhood, association and dialogue established between documents and researchers, as recognized by the scientific community. These two methods have been used individually for the understanding of scientific communication, the research front and intellectual structure of scientific domains. Recently, it has been observed comparative studies between them in order to verify their adequacy and efficiency as indicators of similarity or proximity subject. In this context, due the importance of these methods to the bibliometric and domain analysis, this paper aims to discuss theoretical and conceptual aspects related to both methods of relational citation analysis of relations author co-citation analysis and author bibliographic coupling analysis -, to contribute to the discussions and development of the Brazilian theoretical and methodological studies on the subject.
\end{abstract}

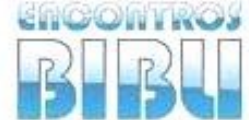

v. 21, n. 47,2016 p. $82-99$

ISSN 1518-2924
Keywords: Co-citation analysis. Bibliographic coupling. Relational citation analysis. Domain analysis. 


\section{INTRODUÇÃo}

Em todo domínio do conhecimento, o ciclo espiral da produção científica tem como premissa o fato de um pesquisador construir seu trabalho sustentado em trabalhos anteriores. Desse modo, o registro da lista de referências em um trabalho científico reflete o diálogo estabelecido pelo pesquisador com seus pares, bem como o processo de construção do seu conhecimento. Assim, ao citar, um pesquisador evidencia quais os referenciais teóricos e metodológicos constituem o paradigma e aporte para o desenvolvimento do seu trabalho.

0 conjunto de referências dos trabalhos científicos pode, nesse contexto, ser analisado como reflexo de uma comunidade científica discursiva, na medida em que explicita tanto o diálogo entre os autores citantes e citados, como o reconhecimento da proximidade e vizinhança teórica e/ou metodológica entre os citados por esta comunidade, retratando assim características do domínio. Alinhado a essa concepção, Macias Chapula (1998) destaca que a citação deve ser pensada um processo social, com a composição das listas de referências que acompanham os trabalhos científicos refletindo características do autor e seu ambiente profissional.

Desse modo, a análise de citação constitui uma abordagem que se caracteriza por sua natureza social, histórica e dinâmica e a sua estreita dependência da literatura acadêmica (HJØRLAND, 2013), que permite identificar os grupos de cientistas, suas publicações, e evidencia os pesquisadores de maior impacto de um domínio (GLÄNZEL, 2003). Aponta seus paradigmas, procedimentos metodológicos pertinentes, bem como os pesquisadores de "vanguarda". Segundo Smiraglia (2011), as citações definem o domínio.

Nesse contexto, a citação é tomada como indicador objetivo da comunicação científica, que evidencia as relações entre documentos e seus autores, tanto citantecitado, como citante-citante e citado-citado na visão do citante.

Diante disso, para se avaliar a interlocução entre os pesquisadores e seu papel nos diferentes domínios científicos, os estudos de citação constituem procedimentos relevantes de análise, na medida em que contribuem para a visualização do processo comunicativo e interativo, bem como da estrutura subjacente do domínio em estudo.

Os estudos de citação e seus indicadores se baseiam em dois tipos de análises: univariáveis ${ }^{1}$ e relacionais. Estas últimas permitem conhecer as relações estruturais de conectividade teórico-metodológica de um domínio, a proximidade, a vizinhança, a associação e a interlocução estabelecida entre os documentos, pesquisadores, periódicos, entre outros, como reconhecidos pela comunidade científica. Para isso, levam em conta a observação simultânea de duas referências, ou

\footnotetext{
1 Os indicadores cientométricos e bibliométricos têm sido classificados como indicadores univariáveis e indicadores relacionais. Nos primeiros, cada elemento em estudo é medido segundo uma dimensão escolhida. Nos últimos, busca-se analisar os elementos segundo medidas de "distância" ou "proximidade" que estimam a "dessemelhança" ou "semelhança" entre os elementos (ROSTAING, 1996), envolvendo assim a observação simultânea de dois indivíduos, como unidade de análise, isto é, baseiam-se sempre em pares (ou ternas, ..., n-úplas) de elementos para a observação da característica (relação) analisada. Callon, Courtial e Penan (1995) denominam os primeiros como indicadores de atividade e consideram que estes seguem um modelo que concebe a ciência como uma atividade produtiva normal, medindo volume de produção e impacto, por meio do cômputo do número de publicações e de citações, por pesquisador, instituição, periódico, país, ano e área, entre outros. Para estes autores, os indicadores relacionais remetem a um modelo diferente de análise do desenvolvimento científico e tecnológico, centrado nas interações na comunidade científica, no qual se privilegia o estudo da dinâmica das múltiplas interações, associações e relações em um domínio científico, por meio da análise das coautorias, das redes de citação e dos estudos de cocitação e da coocorrência de palavras, entre outros.
} 
seja, sustentam-se em medidas que observam a distância ou proximidade entre dois documentos, autores, periódicos, entre outros.

Há dois métodos principais de análise relacional das citações, destinados a mapear as proximidades temáticas, teóricas e/ou metodológicas entre artigos, autores, periódicos, países ou outras unidades de análise: Análise de Acoplamento Bibliográfico e Análise de Cocitação, que, apesar de alguma semelhança, apresentam diferenças importantes (MARSHAKOVA, 1981).

Esses métodos bibliométricos, baseados nas ligações das citações, são usados para a visualização de um domínio, uma vez que buscam revelar domínios da comunicação científica refletidos na literatura científica e nos entrelaçamentos das citações dos pesquisadores em suas publicações (BÖRNER; CHEN; BOYACK, 2003). Entende-se, aqui, a literatura científica como a expressão mais objetiva da representação de um domínio científico, como resultado da atividade social de pesquisa e, neste contexto, o estudo dessa literatura consiste uma forma de análise do domínio.

O Acoplamento Bibliográfico, elaborado por Kessler (1963), mede a relação entre dois artigos com base no número de referências em comum citadas pelos dois artigos. A Análise de Cocitação, por outro lado, mede a relação entre dois artigos com base no número de publicações em que estes aparecem citados concomitantemente. Nesse sentido, Small (1973) apontou que o acoplamento bibliográfico e a análise de cocitação, como indicadores de similaridade temática, fornecem padrões significativamente diferentes em relação à estrutura de um domínio científico.

Nesse contexto, dada a importância do Acoplamento Bibliográfico e da Análise de Cocitação para os estudos bibliométricos e de domínio, este artigo tem por objetivo examinar aspectos teóricos e conceituais inerentes a esses métodos relacionais de citação, presentes na literatura internacional e nacional, visando contribuir com os estudos brasileiros de discussão e aprofundamento da compreensão desta temática.

Justifica-se este artigo em função da carência de estudos brasileiros que tratem da temática proposta. Esta observação decorre do fato de em um levantamento ${ }^{2}$, realizado na base Scopus, para os termos bibliographic coupling e Cocitation analysis or Cocitation analysis, somente 1 dos 120 artigos publicados sobre acoplamento bibliográfico ser de autoria brasileira, de Couto et al (2013), publicado no periódico Informationa Retrieval, em que analisam a contribuição tanto do acoplamento bibliográfico como da análise de cocitação, para fins de classificação automática de documentos. Ainda, do total de 451 artigos publicados com a temática análise de cocitação, somente 8 artigos foram publicados por autores brasileiros, a partir de 2013, todavia, somente 3 em periódicos da Ciência da Informação, a saber, Scientometrics, Scire e Informação \& Sociedade.

Em âmbito da Ciência da Informação no Brasil, o levantamento realizado ${ }^{3}$ na base de dados BRAPCI - Base de Dados Referenciais de Artigos de Periódicos em Ciência da Informação, com o termo acoplamento bibliográfico, recuperou somente um artigo, publicado em 1975, no periódico Ciência da Informação, por Carvalho (1975). Ressalva-se que esta base não apresenta dados atualizados para o ano 2014, em especial em relação ao periódico Em Questão, que publicou um número especial com trabalhos apresentados no IV Encontro Brasileiro de Bibliometria e Cientometria (EBBC) - 2014, entre os quais encontra-se o artigo Lucas e GarciaZorita (2014), que utiliza o método de acoplamento bibliográfico em seu estudo.

\footnotetext{
${ }^{2}$ Realizada em 07 de agosto de 2015.

${ }^{3}$ Em 11 de agosto de 2015.
} 


\section{ACOPLAMENTO BIBLIOGRÁFICO DE AUTORES}

Em 1963, Michael M. Kessler apresentou um método para agrupar artigos científicos e técnicos, baseado em unidades de acoplamento bibliográfico, em que uma unidade de acoplamento entre dois artigos é definida como um item de referência usado pelos dois artigos (KESSLER, 1963). Se há um item de referência em comum entre dois artigos, eles são ditos estarem bibliograficamente acoplados (EGGHE; ROUSSEAU, 2002).

Desse modo, o acoplamento bibliográfico (AB) entre dois artigos ocorre quando estes referenciam pelo menos uma publicação em comum (Figura 1). Nesse contexto, o acoplamento bibliográfico estabelece uma conexão entre dois artigos ao utilizarem as mesmas referências.

A força de acoplamento bibliográfica entre dois artigos não se altera com o tempo, uma vez que a frequência do acoplamento bibliográfico é fixa, não podendo facilmente contribuir para o estudo das mudanças nos domínios científicos ao longo do tempo (SMALL, 1973) e por isso, é considerada uma análise retrospectiva.

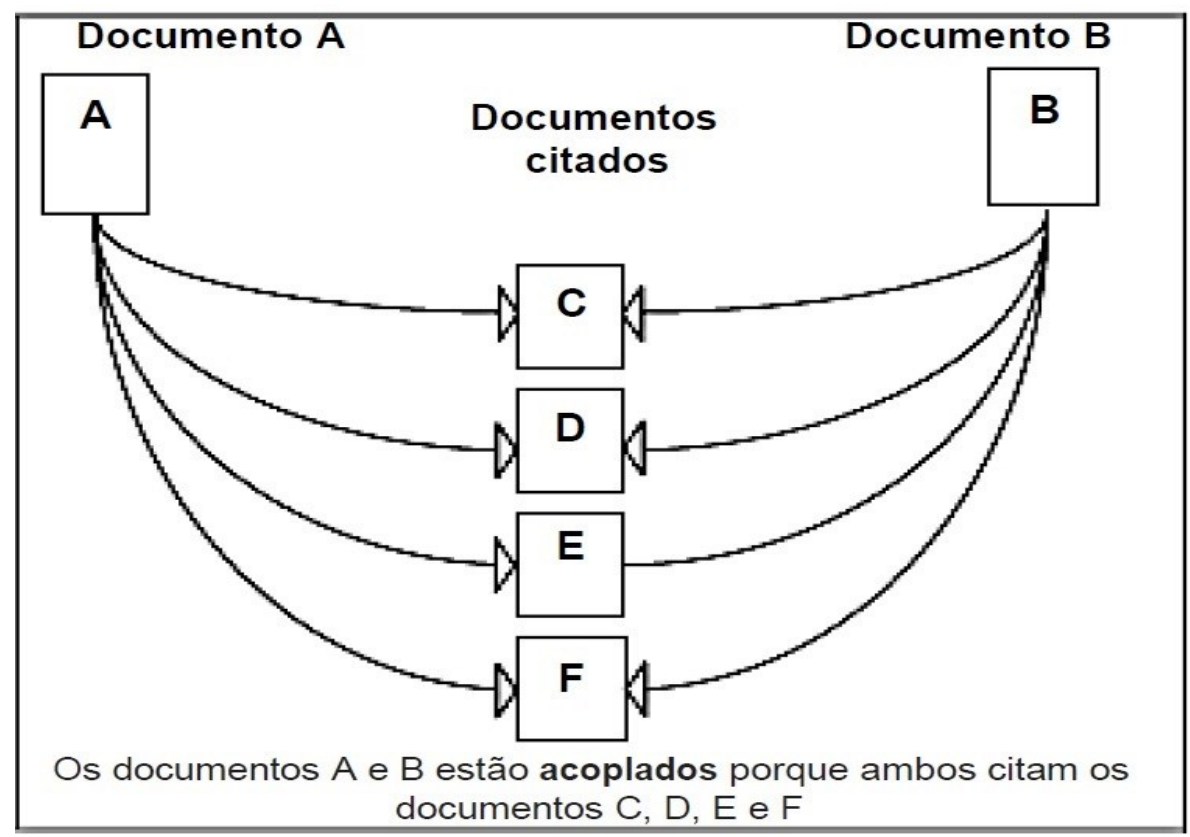

Figura 1: Documentos A e $B$ acoplados bibliograficamente, em função dos quatro documentos C, D, E, F citados, em comum.

Fonte: Mattos e Dias (2010), adaptado de Garfield (2001).

Segundo Kessler (1965), o conjunto de referências utilizado pelos autores em seu artigo evidenciam o ambiente intelectual no qual trabalham e, se dois artigos apresentam bibliografias similares, há uma relação implícita entre eles.

Desse modo, no método de Acoplamento Bibliográfico, parte-se da hipótese de que se dois artigos fazem referência a uma mesma fonte, eles apresentam proximidade teórica e/ou metodológica (KESSLER,1965). Nesse contexto, a intensidade do acoplamento de dois artigos depende da quantidade de referências que eles têm em comum e quanto maior o número de referências em comum, maior será a força de conexão entre eles (EGGHE; ROUSSEAU, 2002, ZHAO; STROTMANN, 2008).

Assim, o Acoplamento Bibliográfico mensura a proximidade entre dois artigos comparando suas referências e quanto maior o número de referências que compartilham, maior a similaridade entre eles, que pode ser temática, teórico, metodológica ou outra particularidade compartilhada (LUCAS; GARCIA-ZORITA; SANZ-CASADO, 2013). 
Nesse contexto, a análise de Acoplamento Bibliográfico possibilita o estudo do desenvolvimento das linhas de pesquisa, permitindo identificar os núcleos de pesquisa, os pesquisadores e os artigos mais importantes em um domínio científico (CARVALHO, 1975). Glänzel (2003) considera este, o método baseado na análise do conjunto de referências mais importante para a visualização de um domínio.

Destaca-se todavia que o método de Acoplamento Bibliográfico (AB), como um indicador de similaridade entre artigos, foi pouco aplicado para a análise da estrutura e rede de conhecimento em um domínio científico, desde a sua introdução na década de 1960, ao contrário da análise de cocitação que, nas duas últimas décadas, se mostra hegemônica como procedimento para a visualização das redes de conhecimento e sua evolução ao longo do tempo (ZHAO; STROTMANN, 2008). No entanto, a partir de meados da década de 2000, observa-se um crescimento nos estudos que analisam as redes de conhecimento geradas com base no método $A B$, como Jarneving $(2005,2007)$, com acoplamento por documentos, e Boyack, Börner e Klavans (2009) com acoplamento de disciplinas, por meio de periódicos.

Zhao e Strotmann (2008), em estudo que estende o método de acoplamento bibliográfico para a conexão entre autores, argumentam que, embora a análise de cocitação de autores seja frequentemente aplicada para mapear a estrutura intelectual de um domínio científico, o que de fato é mapeado nesses estudos é a estrutura das influências intelectuais em um domínio como percebida por seus autores ativos (ZHAO; STROTMANN, 2008).

Nesse contexto, Zhao e Strotmann (2008) introduzem a análise de acoplamento bibliográfico de autores (ABA), como uma ampliação do método proposto por Kessler (1963), a fim de mapear as atividades científicas dos autores e contribuir para a obtenção de uma visualização mais real e aprofundada das estruturas intelectuais de um domínio científico e ampliar a compreensão das análises de redes de citação baseadas nos autores.

No método de acoplamento bibliográfico de autores (ABA), considera-se que quanto mais referências em comum, dois autores têm em suas obras, mais semelhantes são suas investigações (QIU; DONG; YU, 2014).

Segundo Zhao e Strotmann (2008), a análise de acoplamento bibliográfico de autor evita o problema apontado por Small (1973) em relação ao método de Kessler (1963), uma vez que a frequência do acoplamento bibliográfico entre dois autores (isto é, entre suas obras) evolui ao longo do tempo, quando pelo menos um deles continua a publicar. Consideram, ainda, que este método, ao focar nos autores da fonte dos trabalhos (citantes) em lugar dos autores de referências citadas, pode fornecer uma visão mais completa da estrutura, características e desenvolvimento das redes de conhecimento de conhecimento científico.

Segundo os propositores do método, há dois aspectos principais que afetam os resultados da análise ABA: o método de escolha do conjunto de autores para representar o domínio científico estudado e a definição da forma de cômputo da frequência do acoplamento bibliográfico entre os autores.

Em relação à definição do método de cômputo da frequência do acoplamento entre os autores, há duas possibilidades: frequência de acoplamento entre os dois autores citantes ser tomada como o número total de documentos compartilhados em suas listas de referências (Tabela 1); ou de forma mais simples, tratar a obra completa de um autor citado como uma única publicação e calcular a frequência do acoplamento entre os dois autores citantes como o número total de autores compartilhados nas listas de referências das suas publicações (Tabela 2). 
Tabela 1: Ilustração do Acoplamento bibliográfico entre pesquisadores, a partir da frequência de compartilhamento de referências.

\begin{tabular}{|c|c|c|c|c|c|c|c|c|c|c|}
\hline & \multicolumn{10}{|c|}{ Referências (Doc) citadas nas obras dos Autores 1 e 2} \\
\hline $\begin{array}{c}\text { Pesquisadores } \\
\text { Acoplados } \\
\end{array}$ & Doc1 & Doc2 & Doc3 & Doc4 & Doc5 & Doc6 & Doc7 & Doc8 & Doc9 & Doc10 \\
\hline Autor 1 & & $\mathrm{X}$ & $\mathrm{X}$ & & $\mathrm{X}$ & & $\mathrm{X}$ & & $\mathrm{X}$ & $\mathrm{X}$ \\
\hline Autor 2 & $\mathrm{X}$ & $\mathrm{X}$ & & $\mathrm{X}$ & $\mathrm{X}$ & $\mathrm{X}$ & & $\mathrm{X}$ & & \\
\hline
\end{tabular}

Fonte: elaboração própria.

Na tabela 1, os Pesquisadores 1 e 2 são acoplados pelas referências à Doc2 e Doc5. Desse modo, a frequência de acoplamento bibliográfico entre estes pesquisadores é igual a 2 .

Na tabela 2, os pesquisadores 1 e 2 são acoplados pela citação aos autores Autor C, Autor E e Autor F. Desse modo, a frequência de acoplamento bibliográfico entre os pesquisadores 1 e 2 é igual a 3 .

Tabela 2: Ilustração do Acoplamento bibliográfico entre pesquisadores, a partir da frequência de compartilhamento de autores citados.

\begin{tabular}{lccccccc}
\hline & \multicolumn{8}{c}{ Autores citados nas obras dos Autores 1 e 2 } \\
\hline Pesquisadores & Autor & Autor & Autor & Autor & Autor & Autor & Autor \\
Acoplados & A & B & C & D & E & F & G \\
\hline Autor 1 & $\mathrm{X}$ & & $\mathrm{X}$ & $\mathrm{X}$ & $\mathrm{X}$ & $\mathrm{X}$ & \\
Autor 2 & & $\mathrm{X}$ & $\mathrm{X}$ & & $\mathrm{X}$ & $\mathrm{X}$ & $\mathrm{X}$ \\
\hline
\end{tabular}

Fonte: elaboração própria.

Zhao e Strotmann $(2008,2014)$ utilizam em suas análises o Acoplamento bibliográfico entre autores, a partir da frequência de compartilhamento dos autores citados, como ilustrado na tabela 2.

Segundo Hjørland (2013), entender o acoplamento bibliográfico entre autores significa entender o grau de sobreposição da identidade de citação desses autores. Segundo ainda esse autor, essa sobreposição pode ser parcialmente determinada por diferenças entre os diversos domínios científicos, uma vez que em alguns deles, os autores têm grande liberdade na escolha dos problemas de pesquisa, métodos de investigação e também no que considera literatura relevante. Por outro lado, em outros domínios, os pesquisadores estão bastante restringidos pelas normas e convenções desenvolvidas coletivamente. Desse modo, as identidades de citação apresentam maior variabilidade em alguns domínios do que em outros.

A partir da proposta de Zhao e Strotmann (2008), observam-se pesquisas que têm se dedicado a desenvolver o método $\mathrm{ABA}$, em termos teóricos e aplicados. Entre elas, destacam-se: Rousseau (2010) que revisa as noções de acoplamento bibliográfico e cocitação e suas generalizações, assim como as diferentes formas de suas aplicações, como método para evidenciar a estrutura intelectual de um domínio científico; e Ma (2012) que apresenta três métodos para o cálculo da força relativa de acoplamento bibliográfico entre os autores.

Atualmente, alguns pesquisadores têm se dedicado a estender o método de acoplamento de autores a outros tipos de relação de compartilhamento entre os autores, como Cabanac (2011) que mediu o acoplamento entre autores, por meio da similaridade da participação concomitante em conferências científicas (QIU; DONG; YU, 2014). 


\section{ANÁLISE DE COCITAÇÃO DE AUTORES (ACA)}

Henry Small propôs, em 1973, uma forma de analisar a ligação entre dois documentos, baseada no estudo da frequência ${ }^{4}$ com que eles documentos são citados juntos. Denominou essa forma de coocorrência de dois documentos na literatura de cocitação.

Com esse procedimento, Small buscou analisar, identificar e descrever a estrutura e a conectividade de uma área do conhecimento científico (BAYER; SMART; McLAUGHIN, 1990), via documentos publicados.

Ao contrário do acoplamento bibliográfico que liga/assemelha documentos, por meio das suas citações em comum, a cocitação identifica a ligação/semelhança de dois documentos citados, via suas frequências de ocorrência conjunta em uma lista de referências dos autores citantes.

Neste trabalho pioneiro, Small define cocitação como a citação conjunta de dois artigos em uma literatura posterior (Figura 2) e afirma que para dois documentos ser fortemente cocitados, um grande número de autores deve citar os dois trabalhos simultaneamente. Baseado nessa característica da cocitação, o autor destaca que a cocitação é uma relação estabelecida pelos autores citantes (SMALL, 1973).

Segundo ainda o autor, ao se medir a força de cocitação entre dois documentos, se evidencia o grau de associação entre pares de documentos, segundo a compreensão da comunidade de autores citantes, isto é, conforme são reconhecidos pelos citantes (SMALL, 1973). Assim, a proximidade e a interlocução de dois documentos não são determinadas pelos autores dos documentos, mas definidas pela comunidade científica que se apropria do seu conteúdo e estabelece conexões durante o processo de geração de novos conhecimentos.

Desse modo, a intensidade da cocitação entre dois artigos é determinada pelo número de publicações em que ambos os artigos são citados juntos. No momento da publicação, os dois artigos podem parecer não estarem ligados. Suas ligações podem aparecer (e crescer com o tempo), quando estes artigos começam a ser citados conjuntamente na literatura científica. Assim, a força da cocitação é determinada pela reação dos pesquisadores em relação aos artigos publicados (MARSHAKOVA, 1981), ou seja, evidencia a estrutura de conhecimento de uma determinada área, segundo a compreensão da comunidade citante.

A premissa fundamental da análise de cocitação (AC) é que, quando dois ou mais documentos ou autores são citados juntos, em uma pesquisa posterior, há uma proximidade temática, conceitual e/ou metodológica entre os citados, na visão do autor citante (SMIRAGLIA, 2011) e desse modo, quanto maior o número de vezes que dois documentos são citados juntos, mais provável que eles sejam relacionados em conteúdo (BELLARDO, 1980).

Segundo Small (2004), os cientistas definem as estruturas de seus campos científicos ao incluírem em suas listas de referências o que consideram relevante na literatura e esta compreensão da importância é consensual na comunidade. Afirma que a estrutura da ciência é, desse modo, gerada por padrões de correconhecimento, uma vez que ao cocitar dois documentos, os autores citantes estão correconhecendo os citados e criando uma associação de significados.

Alinhado a este pensamento, Hjørland (2002) considera que a Análise de Cocitação mostra detalhes e conexões reais entre pares de documentos. Segundo o autor, essas ligações representam o reconhecimento explícito da dependência entre artigos, pesquisadores, campos, abordagens, entre outros.

\footnotetext{
${ }^{4}$ Embora Small (1973) utilize somente as frequências absolutas de cocitação em sua análise, define frequência relativa de cocitação como a razão entre número de cocitação entre dois documentos e o número total de documentos que citou pelo menos um dos dois documentos (SMALL, 1973, p. 269).
} 
Desse modo, quanto maior a frequência de cocitação, mais próxima a relação entre os citados, sejam documentos, autores, periódicos, países ou temas. A análise de cocitação evidencia a similaridade, complementaridade, sobreposição de ideias entre os autores citados, mas também pode indicar a contraposição destas, no entendimento dos autores citantes.

Nesse contexto, segundo Hjørland (2013), entender os padrões de cocitação significa entender a história do reconhecimento e impacto acadêmico das publicações, uma vez que uma publicação pode ser potencialmente relevante para pesquisas futuras e pode, assim, potencialmente ser citada.

Como proposta por Small (1973), a análise de cocitação ao se sustentar na identificação de artigos basilares e/ou de impacto na comunidade científica e suas ligações, propicia uma visão microscópica da rede de associações, influência e impacto da produção do conhecimento em uma comunidade científica, com autores de maior visibilidade podendo ser representados por vários documentos na rede de cocitações.

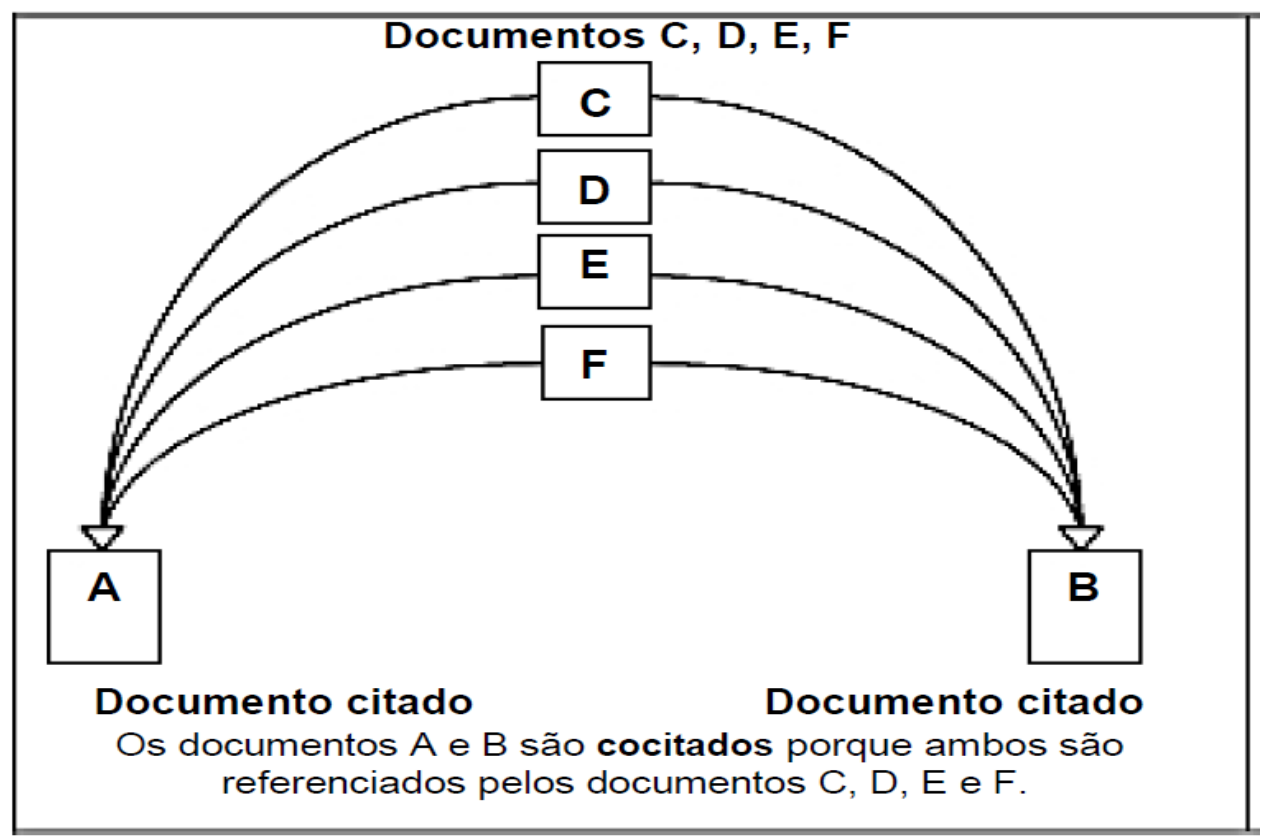

Figura 2: Documentos A e B cocitados, em função dos documentos citantes C, D, E, F. Fonte: Mattos e Dias (2010), adaptado de Garfield (2001).

Nos estudos bibliométricos, conforme propostos por Small (1973), a AC utiliza a análise de redes baseada na coocorrência de artigos científicos nas listas de referências de uma literatura científica para evidenciar e traçar as influências e a evolução das ideias, paradigmas e colégios invisíveis na ciência (BAYER; SMART; MCLAUGHIN, 1990). A partir dos padrões de cocitação de uma literatura pode-se identificar e evidenciar a associação entre conceitos-chave, estabelecidos pela comunidade citante.

Destaca-se, assim, que a AC se embasa em documentos específicos como unidade de análise e na observação de quais pares de documentos são citados juntos na literatura e pode ser usada para estabelecer agrupamentos ou "core" de uma literatura científica.

Apesar da relevância dos trabalhos individuais para mudanças nos paradigmas científicos, o conjunto de publicações de um pesquisador representa melhor a sua influência e seu lugar na estrutura da ciência (BAYER; SMART; MCLAUGHLIN, 1990). 
Sustentados na ideia de que o conjunto da obra de um pesquisador representa mais adequadamente a sua influência e seu lugar na estrutura da ciência que a análise da contribuição individual dos seus artigos, para a evolução teóricoconceitual da sua área do conhecimento, em 1981, White e Griffith propuseram a análise de cocitação baseada no conjunto de contribuições do pesquisador a fim de se visualizar a estrutura intelectual da ciência.

Desse modo, a análise de cocitação de autores (ACA), proposta por White (1981) e White e Griffith (1981), baseia-se na frequência com que um autor está ligado a outro autor, identificada pela citação conjunta de ambos na literatura de uma comunidade científica, sem especificar quais trabalhos dos dois autores foram citados juntos nesta literatura (AHLGREN; HARNEVING; ROUSSEAU, 2003), conforme ilustrado na Figura 3.

Por esta abordagem, assume-se que quanto mais frequentemente dois cientistas são citados juntos e mais semelhantes são seus padrões de cocitação com outros autores, mais estreito o relacionamento entre eles (BAYER; SMART; MCLAUGHLIN, 1990).

Os autores citantes, no desenvolvimento e comunicação de suas pesquisas, estabelecem relações de conteúdo entre os autores citados e criam uma associação de significados desses conteúdos trabalhados, ao fazerem intensa referência simultânea aos autores citados. Assim, a ACA propicia a visualização da estrutural intelectual de uma comunidade científica, evidenciando as associações consolidadas entre os autores citados, na forma como são compreendidas pelos autores citantes dessa comunidade.

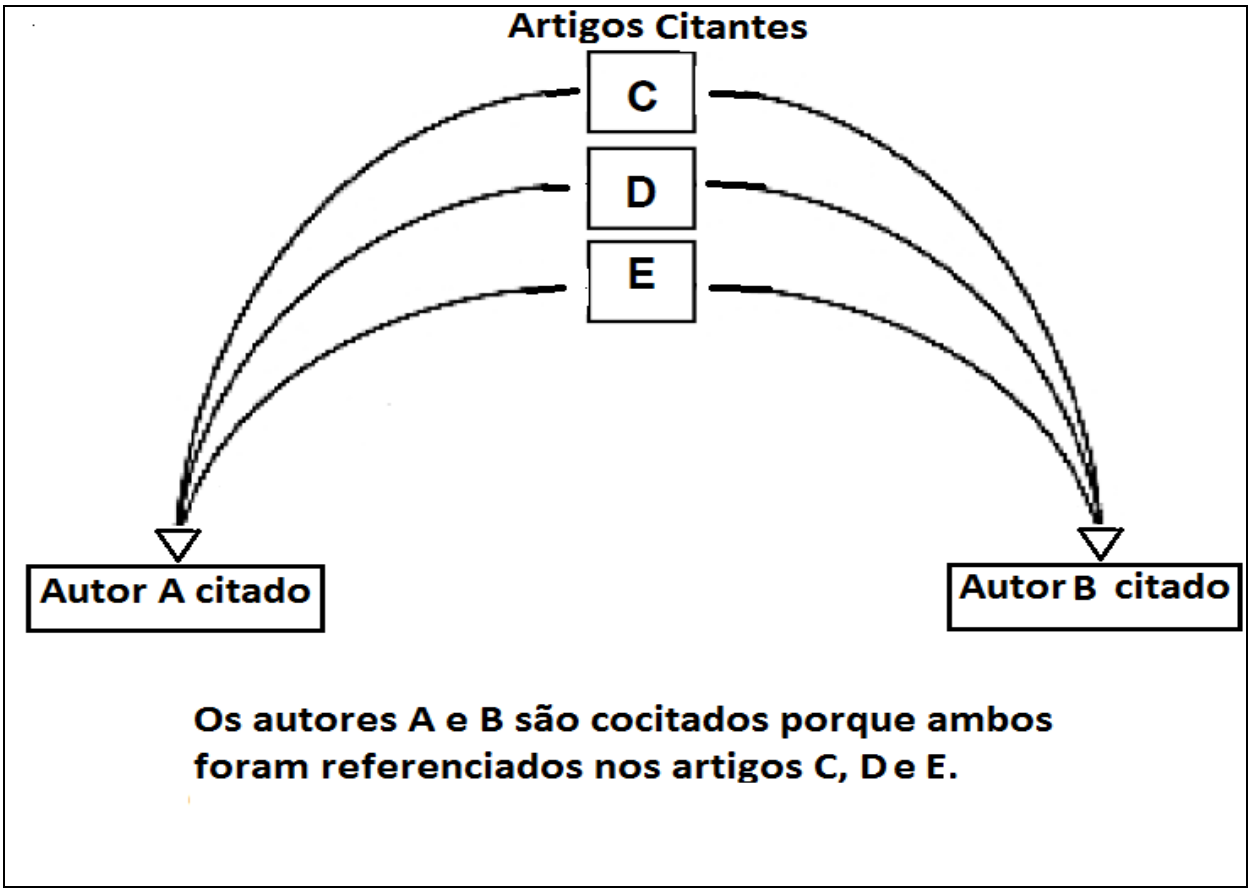

Figura 3: Autores A e B cocitados, em função dos artigos citantes C, D e E. Fonte: elaboração própria, inspirada em Garfield (2001).

Destaca-se, que a proximidade de dois autores detectada por sua alta frequência de cocitação pode ser oriunda de várias situações, entre elas: compartilhamento de referencial teórico e/ou metodológico, similaridade e/ou complementaridade de conteúdos e oposição teórico e/ou metodológica, entre outras. Todavia, quaisquer que sejam as razões para suas citações conjuntas, na percepção dos autores citantes, há uma proximidade de assunto entre os cocitados, seja por similaridade ou contraposição de ideias, por complementaridade teórico- 
metodológica ou compartilhamento metodológico. Ainda, quanto maior a frequência de cocitação, mais consolidado está o correconhecimento estabelecido pelos citantes.

Considera-se, assim, que a principal função da análise de cocitação de autores é identificar os autores influentes em uma área do conhecimento e mostrar suas inter-relações, a partir das citações registradas (WHITE; McCAIN, 1998).

McCain (1990) destaca que a ACA tem como objetivo analisar a estrutura intelectual de um determinado domínio, mostrando sua estrutura social e cognitiva. Segundo a autora, a ACA parte do pressuposto que os pesquisadores, ao citarem fontes similares e próximas, abordam problemas de pesquisa semelhantes na comunidade científica a que pertencem. Nesse sentido, a ACA pode ser definida como análise de um conjunto de autores organizados estruturalmente em rede (social e cognitiva) de um determinado domínio.

Ao apresentar metodologicamente seis passos para o desenvolvimento de uma análise de cocitação de autores McCain (1990) destaca, no sexto passo, a relevância da consulta a especialistas do domínio em estudo, a fim de trazer elementos epistemológicos, teóricos, históricos e sociais para a interpretação e validação do contexto em que os autores foram cocitados (OLIVEIRA; GRÁCIO, 2013). Nesse contexto, reforça-se o paradigma social da ACA, partilhado com a Análise de Domínio, proposta por Hjørland $(1995,2002)$, dado o caráter social, histórico e epistemológico dos motivos para citar, ou não, um autor.

Smiraglia $(2007,2009,2011)$ destaca o importante papel da ACA para a construção de mapas multidimensionais relativos aos parâmetros dos domínios, contribuindo para a visualização do conjunto de temas neles tratados e das suas frente de pesquisa, permitindo a visualização efetiva da intenção e extensão dos domínios. Ainda, a visualização obtida por esse método permite se observar a evolução do conhecimento e da partilha de informação em um domínio, ou entre domínios, assim como identificar seus paradigmas teóricos nucleares e as mudanças neles ocorridas, por tratar com as percepções da comunidade (citante), contribuindo para se identificar, evidenciar e visualizar como essa comunidade reconhece as similaridades entre os pesquisadores (citados) no domínio (SMIRAGLIA, 2007, 2009, 2011).

Alinhado a esse entendimento, Gmür (2003) afirma que a frequência de cocitação entre dois autores citados determina como a estrutura de conhecimento de um domínio é percebida por seus pesquisadores.

Ampliando para outras unidades de análise, Miguel, Moya Anegon e Herreno Solana (2008) afirmam que a análise de cocitação, seja de documentos, autores, jornais, especialidades ou áreas de conhecimento, produz representações válidas da estrutura intelectual de um domínio científico.

A importância da análise de cocitação para a visualização e compreensão das estruturas subjacentes de uma comunidade científica, suscitou estudos teóricos e metodológicos relativos à questão da forma mais adequada do indicador utilizado em sua análise: frequência absoluta ou índice relativo de cocitação. Esses estudos têm mostrado resultados algumas vezes discordantes, seja em defesa da utilização da frequência absoluta, seja em defesa de uma das distintas formas de índices relativos (em especial, Cosseno de Salton, Índice de Jaccard e correlação linear), gerando uma significativa literatura na área da Ciência da Informação.

Alguns autores se posicionaram favoravelmente ao uso dos índices relativos (AHLGREN; HARNEVING; ROUSSEAU, 2003; ECK; WALTMAN, 2008, LEYDESDORFF, 2008). Small e Sweeney (1985) observam que os índices relativos de cocitação proporcionam análises que agrupam os referentes teóricos de forma mais representativa da estrutura intelectual de um domínio científico, ao eliminar a dependência do volume de literatura referenciada, que varia entre as diversas áreas e domínios. 
Leydesdorff e Vaughan (2006) destacam que as frequências absolutas de cocitação contribuem de forma significativa para a construção das redes de cocitação e avaliação da conexão da rede e proximidade científica entre os autores cocitados. Por outro lado, segundo esses autores, os índices relativos de cocitação, associados aos procedimentos multivariados de agrupamento dos autores cocitados, são importantes por mostrar, de forma consistente, aspectos subjacentes da estrutura intelectual de um domínio científico, não identificáveis nas análises de cocitação somente com frequências absolutas.

Luukkonen et al. (1993) destacam a relevância de usar os índices absolutos e relativos nas análises relacionais entre autores, uma vez que cada um deles evidencia um aspecto diferente da estrutura de um domínio. Os índices absolutos expõem características relativas à centralidade dos autores e periferia nas redes, ao passo que os índices relativos explicitam a intensidade das relações entre os pares de autores.

No Brasil, alguns pesquisadores também têm tratado das questões relativas à adequação e aspectos representados pelos índices absolutos e relativos de cocitação, para a visualização da estrutura de um domínio científico. Destacam-se os estudos de Mattos e Dias (2010) e Grácio e Oliveira (2013, 2014 e 2015). Os primeiros apresentam pesquisa teórico-metodológica relativa ao debate existente na literatura científica da área acerca da obtenção das frequências de cocitação, construção e transformação destas em matrizes de proximidade e a escolha da medida de proximidade (absoluta ou relativa).

Em um estudo também de natureza teórico-metodológica Grácio e Oliveira (2013) realizam uma análise comparativa da contribuição dos indicadores de proximidade absolutos e relativos - Cosseno de Salton e Índice de Jaccard-, para a análise de cocitação de autores, em que concluem que os índices relativos diferenciaram a intensidade de proximidade entre os autores não discriminada por frequências absolutas, em função da presença de cada autor no domínio estudado.

Em artigo subsequente, as autoras analisam a contribuição dos indicadores de proximidade absoluto e normalizado pelo Cosseno de Salton, para a análise de cocitação de autores, e apresentam a integração dos resultados relativos às proximidades reveladas em uma representação visual (rede) que reúne as informações advindas das duas medidas, visando contribuir para a melhor compreensão de um domínio do conhecimento científico (GRÁCIO; OLIVEIRA, 2014).

Em continuidade, Grácio e Oliveira (2015) analisam e comparam os resultados obtidos para a ACA, utilizando duas medidas relativas de proximidade entre autores - Coeficiente de Correlação de Pearson (r), considerada medida global de proximidade, e Cosseno de Salton (CS), medida local de proximidade - em que constatam que tendências semelhantes ocorrem para baixa proximidade local e baixa proximidade global.

\section{ESTUDOS COMPARATIVOS ENTRE ACA E ABA}

A partir de um levantamento ${ }^{5}$ realizado na base Scopus, com o termo de busca bibliographic coupling, recuperou-se um total de 120 artigos publicados, em especial, por autores dos Estados Unidos (25), da China (12), de Taiwan (12), Alemanha (11) e Espanha (8) e socializados, mormente, nos periódicos: Scientometrics (34), Journal of Informetrics (9) e Journal of the American Society for Information Science and Technology (8).

Nesse levantamento, observou-se também que, embora o primeiro artigo sobre Acoplamento Bibliográfico date de 1963, até o início da década de 1990,

\footnotetext{
${ }^{5}$ Realizada em 07 de agosto de 2015.
} 
somente $10 \%$ desse conjunto de artigos tinha sido publicado. No período entre 1993 e 2008 (16 anos), publicaram-se outros 28\% desse conjunto de artigos. Assim, os estudos sobre acoplamento bibliográfico têm sido mais frequentes nos últimos 7 anos (2009 a 2015), uma vez que a maioria (62\%) dos artigos recuperados, datam deste período.

O levantamento realizado na base Scopus, na mesma data, com os termos de busca Co-citation analysis or Cocitation analysis recuperou um total de 451 artigos, evidenciando que este método têm sido mais usado para se avaliar um domínio científico, em relação ao Acoplamento Bibliográfico, e corroborando observação de Glänzel (2003) e Boyack e Klavans (2010).

Para este conjunto de publicações, embora mais volumoso que o recuperado para a temática Acoplamento Bibliográfico, observaram-se tendências temporais similares, assim como de autoria e principais canais de comunicação. Em relação à autoria, a temática tem sido desenvolvida, em especial, por autores dos Estados Unidos (125), China (65), Canadá (37), Reino Unido (33), Espanha (30), Alemanha (29) e Taiwan (24) e socializados, principalmente, nos periódicos: Scientometrics (91), Journal of the American Society for Information Science and Technology (45), Information Processing and Management (11) e Journal of Informetrics (10).

Em termos temporais, a temática também tem seu maior volume de publicações em anos mais recentes: $12 \%$ dos artigos publicados até o início da década de 1990; 33\% publicado no período de 1993 a 2008; e 55\% dos artigos foram publicados nos últimos 7 anos (2009-2015).

Embora os métodos de acoplamento bibliográfico e de cocitação sejam, atualmente, usados com frequência nas pesquisas relativas às frentes de pesquisa $\mathrm{e}$ às estruturas sociais e cognitivas de um domínio científico, a maioria emprega apenas um dos dois métodos e raramente os integram em suas investigações (HUANG; CHANG, 2015). Grácio e Oliveira $(2013,2014)$ citam diversos estudos de análise de cocitação, em âmbito internacional e nacional, e Huang e Chang (2015) mencionam aplicações do acoplamento bibliográfico, em âmbito internacional.

Recentemente, pesquisadores da área observaram a necessidade de estudos que analisem, de forma comparativa, esses dois métodos relacionais de análise de citação. Na última década, observam-se estudos comparativos entre o acoplamento bibliográfico e análise de cocitação, a fim de saber se estes são indicadores adequados e eficientes de similaridade ou proximidade temática. Entre esses estudos, destacam-se Jarneving (2005), Zhao e Strotmann (2008), Boyack e Klavans (2010), Lu e Wolfram (2012) e Qiu, Dong e Yu (2014).

A análise de Jarneving (2005), comparando artigos bibliograficamente acoplado com artigos cocitados, conclui que a frente de pesquisa é retratada de formas distintas, de acordo com o método empregado.

Em estudo analisando a contribuição da Análise de Cocitação de Autores e do Acoplamento Bibliográfico de Autores para a visualização da estrutura do domínio científico da Ciência da Informação, Zhao e Strotmann (2008) concluem que os dois métodos se complementam e que, quando combinados, fornecem uma visualização mais abrangente e real da estrutura intelectual do domínio, permitindo observar a sua trajetória evolutiva, uma vez que o método ABA fornece uma imagem da estrutura em termos da atividade de pesquisa em curso e a ACA fornece uma imagem das influências intelectuais sobre as atividades de pesquisa.

Entre os resultados obtidos por Boyack e Klavans (2010), em estudo comparativo sobre a precisão de quatro métodos distintos para mapeamento do domínio da Biomedicina, entre eles o Acoplamento Bibliográfico e a Análise de Cocitação, conclui que cada método pode ser considerado uma forma de representar a frente de pesquisa no domínio estudado e aponta que, entre os métodos relacionais de citação, o acoplamento bibliográfico apresenta resultados 
ligeiramente superiores que a análise de cocitação para a visualização da estrutura científica do domínio estudado.

No estudo comparativo entre cinco medidas relacionais em nível micro (autores), aplicado à Ciência da Informação, entre elas, análise de cocitação e acoplamento bibliográfico, Lu e Wolfram (2012) concluem que cada método apresenta perspectivas e propriedades diferentes do domínio estudado.

Em estudo para examinar a contribuição de cinco tipos de redes de coocorrência de autores, entre elas acoplamento bibliográfico e análise de cocitação de autores, para a análise da comunicação científica e da estrutura intelectual do domínio da Ciência da Informação, Qiu, Dong e Yu (2014) concluíram que o Acoplamento mostra vantagem significativa ao revelar estrutura científica com mais alta precisão e exatidão, além de identificar mais subestruturas que os outros tipos de rede e que a rede de cocitação de autores tem capacidade moderada para revelar a estrutura de um domínio científico.

De forma sintética e ilustrativa, a Figura 4 exibe, simultaneamente, as relações observadas pelo Acoplamento Bibliográfico de Autores (ABA) e pela Análise de Cocitação de Autores (ACA) para a caracterização da interlocução entre autores e da estrutura subjacente a um domínio científico, identificando e evidenciando as conexões sobre as quais se constrói e socializa o conhecimento.

Alguns estudiosos apontam que ao conectar artigos que cita(m) a(s) mesma(s) publicações(s), o acoplamento bibliográfico constitui uma análise com característica retrospectiva (sincrônica), ao passo que a cocitação, ao conectar os artigos quando aparecem citados concomitantemente na literatura do domínio científico, configura uma análise com característica prospectiva, ou diacrônica (MARSHAKOVA, 1981, GARFIELD, 2001, LUCAS; GARCIA-ZORITA, 2014).

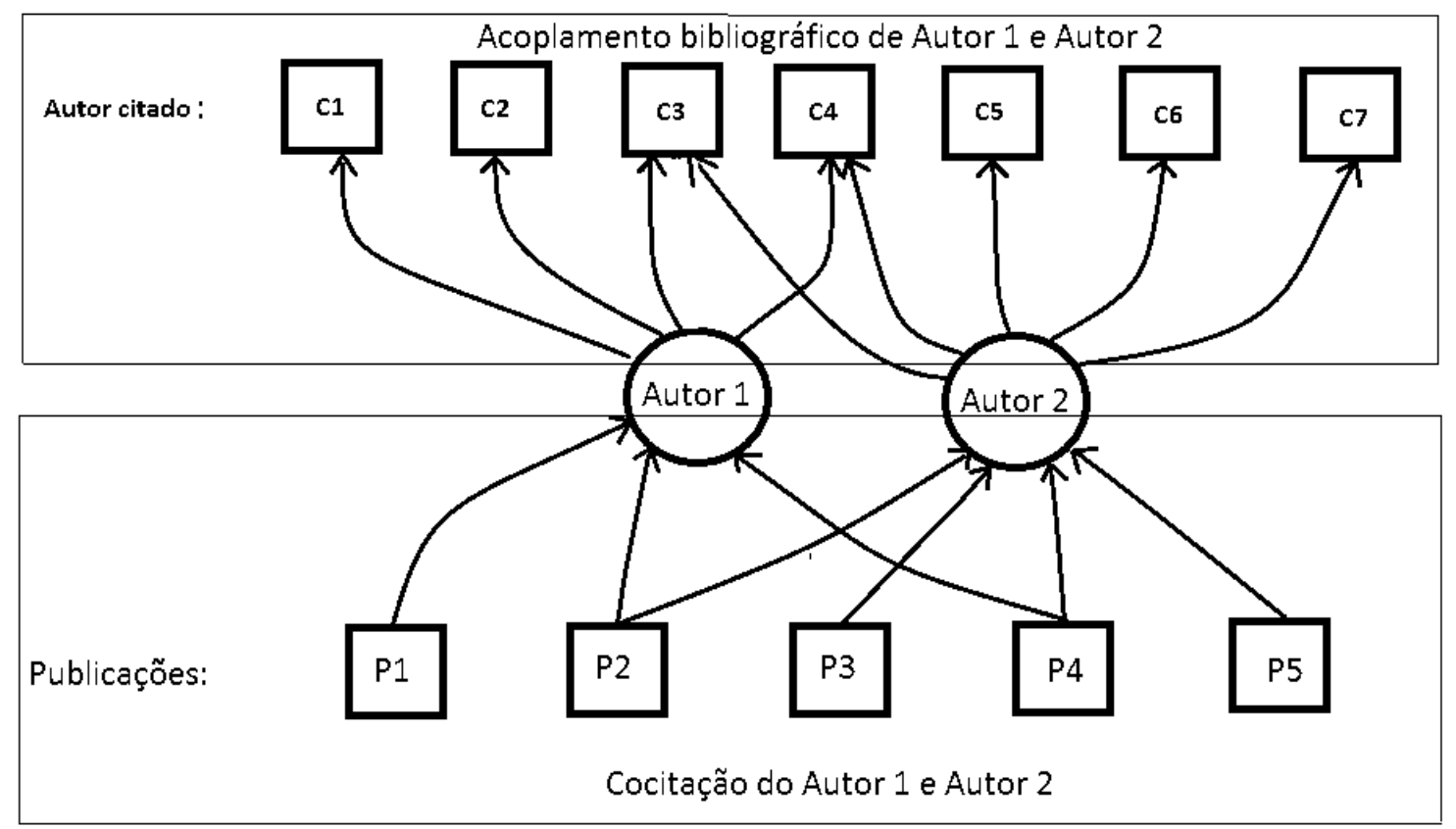

Acoplamento bibliográfico: Autor 1 e Autor 2 estão bibliograficamente acoplados pelos autores citados C3 e C4.

Cocitacão de autores: Autor 1 e Autor 2 são cocitados pelas publicacõas P2 e P4.

Figura 4: Ilustração comparativa do procedimento de análise entre Acoplamento Bibliográfico de Autores e Análise de Cocitação de Autores.

Fonte: elaboração própria.

Em decorrência do tempo necessário para o reconhecimento dos autores em um domínio científico, o acoplamento bibliográfico pode oferecer visualizações mais 
precisas nos estágios iniciais de desenvolvimento de um domínio, quando comparado à cocitação. Ainda, a análise de cocitação restringe a análise aos artigos mais frequentemente utilizados, ao passo que o acoplamento bibliográfico se estende a praticamente todas as publicações referenciadas (GLÄNZEL, 2003).

Os dois tipos de análise relacional de citações têm sido utilizados para diversas questões consignadas à Ciência da Informação, como para a compreensão da comunicação científica, a frente de pesquisa e a estrutura intelectual de um domínio científico.

As análises relacionais de citação em nível micro (coocorrência de autor) têm prevalecido na análise da comunicação e das estruturas intelectuais de um domínio científico, uma vez que, nos outros níveis de agregação (revistas, instituições, países, entre outros), as redes são decorrentes das relações acadêmicas dos autores, como as relações de colaboração ou de citação realizadas pelos autores (QIU, DONG, YU, 2014).

\section{CONSIDERAÇÕES FINAIS}

Este estudo se propôs a contribuir com o desenvolvimento teóricometodológico dos Estudos Métricos da informação, especialmente da temática análise relacional de citação, com foco nos métodos de acoplamento bibliográfico de autores e de cocitação de autores, evidenciando suas premissas, conceitos, diferenças e similaridades, a fim de cooperar para a compreensão dos aspectos teórico e metodológicos que os definem. Ainda, buscou comparar as características por eles mensuradas na visualização da comunicação científica e da estrutura intelectual de um domínio.

Observou-se que o método de acoplamento bibliográfico, ao analisar as referências e autores compartilhados por dois cientistas na construção das suas pesquisas, permite identificar a sobreposição da identidade teórico e/ou metodológica desses cientistas, assim como visualizar a estrutura intelectual, social e cognitiva na perspectiva deles próprios. Evidencia, ainda, as similaridades de ambiente científico em que atuam os pesquisadores analisados.

Considera-se, assim, que o acoplamento bibliográfico $\mathrm{AB}$ permite identificar as correntes teóricas, e seus pesquisadores componentes, em um dado momento do desenvolvimento de um domínio científico, com significativa contribuição para a visualização da estrutura intelectual e correntes que vigoram em um domínio em estudo, nos estágios iniciais do seu desenvolvimento.

Por outro lado, observou-se que a Análise de Cocitação de Autores, ao examinar a proximidade de dois pesquisadores pela frequência com que são citados de forma conjunta pela comunidade científica, evidencia o reconhecimento da estrutura intelectual e social construída pela comunidade científica, de forma independente da percepção dos próprios cocitados.

Desse modo, a intensidade da proximidade dos autores citados é determinada pela reação da comunidade científica, que define, desse modo, a estrutura do seu campo científico. Considera-se, assim, que a análise de cocitação de autores contribui para a compreensão da história do correconhecimento e impacto acadêmico dos pesquisadores em um domínio científico.

Considera-se que compreender os conceitos subjacentes aos métodos de acoplamento bibliográfico e de cocitação contribui para a melhor aplicação de ambos, dado que mensuram características distintas de um domínio, com o acoplamento bibliográfico focando nos grupos de autores citantes e a cocitação focando nos pares de artigos e autores citados na literatura. Assim, reiterando Hjørland (2013), não se pode considerar um deles superior ao outro em todas as situações. 
Nesse contexto, aprecia-se que podem ser considerados métodos complementares, os quais, quando utilizados de forma conjunta, permitem uma visualização mais ampla, real e precisa da dinâmica da estrutura intelectual, social e cognitiva de um domínio científico.

Apesar da relevância dos dois métodos para a visualização das estruturas e redes de conhecimento de um domínio científico, constatou-se que o método de Acoplamento Bibliográfico, embora proposto anteriormente à Análise de cocitação, foi pouco aplicado nos estudos bibliométricos em âmbito internacional e brasileiro, com a presença hegemônica da ACA nas duas últimas décadas. Esse comportamento parece vir se modificando a partir de meados da década de 2000, com um incremento dos estudos baseados no método ABA.

Finalizando, considerando os poucos estudos bibliométricos brasileiros relativos a questões teórico-conceituais das análises relacionais de citação, em especial referentes aos métodos de acoplamento bibliográfico de autores e de cocitação de autores, espera-se contribuir para a ampliação da compreensão destes, na busca do desenvolvimento dos procedimentos adequados para o contexto da ciência brasileira, no sentido de propiciarem a melhor visualização da estrutura intelectual do domínio científico.

\section{REFERÊNCIAS}

AHLGREN, P.; JARNEVING, B.; ROUSSEAU, R. Requirements for a cocitation similarity measure, with special reference to Pearson's correlation coefficient. Journal of the American Society for Information Science and Technology, v. 54, n. 6, p.550-560, 2003.

BAYER, A. E.; SMART, J. C.; McCLAUGHLIN, G. W. Mapping intellectual structure of a scientific subfield through author cocitations. Journal of the American Society for Information Science, v. 41, n. 6, p.444 - 452, 1990.

BELLARDO, T. The use of co-citations to study science. Library Research, v.2, n.3, p. 231$237,1980$.

BÖRNER, K.; CHEN, C.; BOYACK, K. W. Visualizing knowledge domains. Annual Review of Information Science and Technology, v. 37, n. 1, p. 179-255, 2003.

BOYACK, K.; BÖRNER, K.; KLAVANS, R. Mapping the structure and evolution of chemistry research. Scientometrics, v. 79, n.1, p. 45-60, 2009.

BOYACK, K. W.; KLAVANS, R. Co-citation analysis, bibliographic coupling, and direct citation: Which citation approach represents the research front most accurately?. Journal of the American Society for Information Science and Technology, v. 61, n. 12, p. 2389-2404, 2010.

CABANAC, G. Accuracy of inter-researcher similarity measures based on topical and social clues. Scientometrics, v. 87, n.3, p. 597-620, 2011.

CALLON, M.; COURTIAL, J-P.; PENAN, H. Cienciometría - La medición de la actividad científica: de la bibliometría a la vigilância tecnológica. Asturias: Ediciones Trea, S.L., 1995.

CARVALHO, M.M. Análise bibliométrica da literatura de Química no Brasil. Ciência da Informação, v. 4, n. 2, 119-141, 1975. 
COUTO, T. et al. Classifying documents with link-based bibliometric measures. Information Retrieval, v. 13, n. 4, p. 315-345, 2010.

ECK, N. J. V.; WALTMAN, L. Appropriate similarity measures for author co-citation analysis. Journal of the American Society for Information Science and Technology, v. 59, n. 10, p. 1653-1661, 2008.

EGGHE, L.; ROUSSEAU, R. Co-citation, bibliographic coupling and a characterization of lattice citation networks. Scientometrics, v. 55, n. 3, p. 349-361, 2002.

GARFIELD, E. From Bibliographic Coupling to Co-Citation Analysis via Algorithmic HistorioBibliography A Citationist's Tribute to Belver C. Griffith. Conferência ministrada em Drexel University, Philadelphia. 2001 Disponível em: http://garfield.library.upenn.edu/papers/drexelbelvergriffith92001.pdf. Acesso em: 02 de julho de 2015.

GLÄNZEL, W. Bibliometrics as a research field: a course on theory and application of bibliometric indicators. Bélgica, 2003. Disponível em: http://citeseerx.ist.psu.edu/viewdoc/download?doi=10.1.1.97.5311\&rep=rep1\&type=pdf. Acesso em: 9 de janeiro de 2011.

GMÜR, M. Co-citation analysis and the search for invisible colleges: A methodological evaluation. Scientometrics, v. 57, n. 1, p. 27-57, 2003.

GRÁCIO, M. C. C.; OLIVEIRA, E. F. T. Análise de cocitação de autores: um estudo teóricometodológico dos indicadores de proximidade, aplicados ao GT7 da ANCIB. Liinc em Revista, v. 9, n. 1, p. 196-213, 2013.

GRÁCIO, M. C. C.; OLIVEIRA, E. F. T. Estudos de Análise de Cocitação de Autores: uma abordagem teórico-metodológica para a compreensão de um domínio. Tendências da Pesquisa Brasileira em Ciência da Informação, v. 7, n. 1, p. 1-22, 2014.

GRÁCIO, M. C. C.; OLIVEIRA, E. F. T. Indicadores de proximidades em Análise de Cocitação de Autores: um estudo comparativo entre Coeficiente de Correlação de Pearson e Cosseno de Salton. Informação \& Sociedade, v. 25, n.2, p. 105-116, 2015.

HJØRLAND, B.; ALBRECHTSEN, H. Toward a new horizon in Information Science: DomainAnalysis. Journal of the American Society for Information Science, v.6, n. 6, p. 400-425, 1995.

HJØRLAND, B. Domain analysis in information science: eleven approaches-traditional as well as innovative. Journal of Documentation, v.58, n.4, p. 422-462, 2002.

HJØRLAND, B. Citation analysis: A social and dynamic approach to knowledge organization. Information Processing and Management, v. 49, n. 6, p. 1313-1325, 2013.

HUANG, M.-H.; CHANG, C.-P. A comparative study on detecting research fronts in the organic light-emitting diode (OLED) field using bibliographic coupling and co-citation.

Scientometrics, v. 102, n. 3, p. 2014-2057, 2015.

JARVENING, B. A comparison of two bibliometric methods for mapping of the research front. Scientometrics, v. 65, n. 2, p. 245-263, 2005.

JARVENING, B. Bibliographic coupling and its application to research-front and other core documents. Journal of Informetrics, v. 1, n. 4, p. 287-307, 2007.

KESSLER, M. M. Bibliographic coupling between scientific papers. American Documentation, v. 14, n. 1, p. 10-25, 1963. 
KESSLER, M. M. Comparison of the results of bibliographic coupling and analytic subject indexing. American Documentation, v. 16, n.3, p. 223-233, 1965.

LEYDESDORFF, L. On the normalization and visualization of author co-citation data: Salton's cosine versus the Jaccard index. Journal of the American Society for Information Science and Technology, v. 59, n. 1, p.77-85, 2008.

LEYDESDORFF, L.; VAUGHAN, L. Co-occurrence Matrices and their applications in Information Science: Extending ACA to the Web environment. Journal of the American Society for Information Science and Technology, v. 57, n. 12, p.1616-1628, 2006.

LU, K.; WOLFRAM, D. Measuring author research relatedness: A comparison of word-based, topic-based, and author cocitation approaches. Journal of the American Society for Information Science and Technology, v. 63, n.10, p. 1973-1986, 2012.

LUCAS, E. O.; GARCIA-ZORITA, J. C.; SANZ-CASADO, E. Evolução histórica de investigação em informetria: ponto de vista espanhol. Liinc em Revista, v. 9, n. 1, p. 255-270, 2013.

LUCAS, E. O.; GARCIA-ZORITA, C. Produção Científica sobre Capital Social:estudo por acoplamento bibliográfico. Em Questão, v. 20, n. 3, ed. esp., p. 27-42, 2014.

LUUKKONEN, T. et al. The measurement of international scientific collaboration. Scientometrics, v. 28, n. 1, p. 15-36, 1993.

MA, R. Author bibliographic coupling analysis: a test based on a Chinese academic database. Journal of Informetrics, v. 6, n.4, p. 532-542, 2012.

MACIAS-CHAPULA, C. A. O papel da informetria e da cienciometria e sua perspectiva nacional e internacional. Ciência da Informação, v.27, n.2, p.134-40, 1998.

McCAIN, K. Mapping author intellectual space: a technical overview. Journal o the American Society for Information Science. v.41, n.66, p.433-443, 1990.

MARSHAKOVA, I.V. Citation networks in Information Science. Scientometrics, v. 3, n. 1, p. 13-26, 1981.

MATTOS, A.M.; DIAS, E.W. Análise de Cocitação de Autores: questões metodológicas. IN: XI Encontro Nacional de Pesquisa em Ciência da Informação: Inovação e inclusão social: questões contemporâneas da informação, Rio de Janeiro, 2010. Anais..., 2010.

MIGUEL, S.; MOYA-ANEGON, F.; HERRERO-SOLANA, V. A new approach to institutional domain analysis: Multilevel research fronts structure. Scientometrics, v. 74, n.3. p. 331-344, 2008.

OLIVEIRA, E.F.T; GRÁCIO, M.C.C. Studies of Author Cocitation Analysis: A Bibliometric Approach for Domain Analysis. Iris, v.2, n.1, p.12-23, 2013.

QIU, J. P.; DONG, K.; YU, H-Q. Comparative study on structure and correlation among author co-occurrence networks in bibliometrics. Scientometrics, v. 101, p. 1345-1360, 2014.

ROUSSEAU, R. Bibliographic coupling and co-citation as dual notions. A Festschrift in Honour of Peter Ingwersen. e-Zine of the ISSI, v. esp., p. 173-183, 2010.

ROSTAING, H. La bibliométrie e ses techniques. Tolouse: Sciences de la Société, 1996.

SMALL, H. Cocitation in the scientific literature: A new measure of the relationship between two documents. Journal of the American Society for Information Science, v. 24, n. 4, p. 265-269, 1973. 
SMALL, H. On the shoulders of Robert Merton: towards a normative theory of citation. Scientometrics, v. 60, n.1, p. 71-79, 2004.

SMALL, H.; SWEENEY, E. Clustering the Science Citation Index using co-citations: 1. A comparison of methods. Scientometrics, v. 7, n. 3-6, p.391-409, 1985.

SMIRAGLIA, R.P. A glimpse at Knowledge Organization in North America: an editorial. Knowledge Organization, v. 34, n. 2, p.69-71, 2007.

SMIRAGLIA, R.P. Modulation and Specialization in North American Knowledge Organization: visualizing pioneers. In: Proceedings form North American Symposium on Knowledge Organization, v. 2, p. 35-46, 2009.

SMIRAGLIA, R.P. ISKO 11's Diverse Bookshelf: an editorial. Knowledge Organization, v. 38, n. 3 , p. 179-186, 2011.

WHITE, H. D. Cocited author retrieval online: an experiment with the social indicators literature. Journal of the American Society for Information Science and Technology, v. 32 , n. 1, p. 16-21, 1981.

WHITE, H. D.; GRIFFITH, B. C. Author co-citation: a literature measure of intellectual structure. Journal of the American Society for Information Science and Technology, v. 32, n. 3, p.163-171, 1981.

WHITE, H.D.; MCCAIN, K.W. Visualizing a discipline: an author co-citation analysis of Information Science, 1972-1995. Journal of the American Society for Information Science, v. 49, n. 4, p. 327-355, 1998.

ZHAO, D.; STROTMANN, A. Evolution of Research Activities and Intellectual Influences in Information Science 1996-2005: Introducing Author Bibliographic-Coupling Analysis. Journal of the American Society for Information Science and Tecnhology, v. 59, n. 13, p. 2070-2086, 2008.

ZHAO, D.; STROTMANN, A. The Knowledge Base and Research Front of Information Science 2006-2010: An Author Cocitation and Bibliographic Coupling Analysis. Journal of the American Society for Information Science and Tecnhology, v. 65, n. 5, p. 995-1006, 2014. 